\title{
Gut microbiota metagenomics in aquaculture: factors influencing gut microbiome and its physiological role in fish
}

\begin{abstract}
Fish gut microbiome confers various effects to the host fish; this includes overall size, metabolism, feeding behaviour and immune response in the fish. The emergence of antimicrobial-resistant (AMR) bacteria and hard to cure fish diseases warrant the possible utilization of gut microbes that exhibits a positive effect on the fish and thus lead to the usage of these microbes as probiotics. The widespread and systematic use of antibiotics has led to severe biological and ecological problems, especially the development of antibiotic resistance that affects the gut microbiota of aquatic organisms. Probiotics are proposed as an effective and environmentally friendly alternative to antibiotics, known as beneficial microbes. At the same time, prebiotics are considered beneficial to the host's health and growth by decreasing the prevalence of intestinal pathogens and/or changing the development of bacterial metabolites related to health. Uprise of sequencing technology and the development of intricate bioinformatics tools has provided a way to study these gut microbes through metagenomic analysis. From various metagenomic studies, ample of information was obtained; such information includes the effect of the gut microbiome on the physiology of fish, gut microbe composition of different fish, factors affecting the gut microbial composition of the fish and the immunological effect of gut microbes in fish; such this information related to the fish gut microbiome, their function and their importance in aquaculture is discussed in this review.
\end{abstract}

